\title{
Phylogenetic analysis of combined lobular and ductal carcinoma of the breast
}

\author{
HIROKO KOBAYASHI $^{1}$, TOKIKO NAKAI ${ }^{2}$, YOKO NAKANISHI $^{1}$, MARIKO ESUMI $^{3}$ and SHINOBU MASUDA $^{1}$ \\ ${ }^{1}$ Division of Oncologic Pathology, Department of Pathology and Microbiology, Nihon University School of Medicine, \\ Tokyo 173-8610; ${ }^{2}$ Department of Pathology and Clinical Laboratories, National Cancer Center Hospital East, \\ Kashiwa, Chiba 277-8577; ${ }^{3}$ Division of Biochemistry, Department of Biomedical Sciences, \\ Nihon University School of Medicine, Tokyo 173-8610, Japan
}

Received October 24, 2020; Accepted July 12, 2021

DOI: $10.3892 / \mathrm{mmr} .2021 .12357$

\begin{abstract}
Breast cancer manifests in diverse forms, with particular reference to various cell types harboring different mutations and gene expression profiles. To elucidate the clonal relationship between cancer cells in tumors composed of both ductal and lobular phenotypes, two combined lobular and ductal carcinoma (CLDC) cases were analyzed, including one mixed ductal-lobular carcinoma (MDL) lesion, by direct sequencing of the mitochondrial DNA D-loop, digital PCR targeting of chromosomes $1 \mathrm{q}$ and $16 \mathrm{q}$, as well as next-generation sequencing. DNA was extracted from formalin-fixed paraffin-embedded tissue sections of different histological types, including invasive ductal carcinoma, invasive lobular carcinoma, ductal carcinoma in situ, lobular carcinoma in situ, flat epithelial atypia, non-neoplastic mammary gland and extramammary organs, using laser-assisted microdissection. Mutations detected by the comprehensive cancer panel were
\end{abstract}

Correspondence to: Professor Shinobu Masuda, Division of Oncologic Pathology, Department of Pathology and Microbiology, Nihon University School of Medicine, Ohyaguchikami-cho 30-1, Tokyo 173-8610, Japan

E-mail: masuda.shinobu@nihon-u.ac.jp

Abbreviations: EMNT, extramammary non-neoplastic tissue; CCP, comprehensive cancer panel; CLDC, combined lobular and ductal carcinoma; CNV, copy number variation; DCIS, ductal carcinoma in situ; FEA, flat epithelial atypia; FFPE, formalin-fixed paraffin-embedded; GOV, gain of variation; HV, hypervariable region; IDC, invasive ductal carcinoma; ILC, invasive lobular carcinoma; LCIS, lobular carcinoma in situ; LMD, laser microdissection; LN, lymph node; LOH, loss of heterozygosity; LOV, loss of variation; MDL, mixed ductal-lobular carcinoma; mtDNA, mitochondrial DNA; NB, normal breast; qPCR, quantitative PCR; SNP, single nucleotide polymorphism; SNV, single nucleotide variant; SSRNV, simple sequence repeat number variation

Key words: combined lobular and ductal carcinoma, phylogenetic analysis, phenotype-genotype correlation, low-grade breast cancer, precancerous lesion validated by SYBR green allele-specific quantitative PCR (RRM1, AKT1, PIK3CA, RALGDS, EGFR, TP53, IL21R, DPYD, SGK1, CDH1, TIMP3 and KMT2C). CLDC, which shared the basic genetic alterations of $1 \mathrm{q}$ gain or $16 \mathrm{q}$ loss, progresses to invasive lobular or ductual carcinoma with the accumulation of further mutations. Cancer cells contained in an MDL lesion shared closely related genetic alterations, suggesting that these cells have the same origin, despite different histological features, namely 'lobular' or 'ductal'. By contrast, multiple lesions located away from the main tumor, diagnosed as CLDC (excluding an MDL lesion) were not always identical with different genetic alterations, despite being diagnosed as ductal carcinoma in situ. Thus, MDL should be defined as a distinct category separate from CLDC, whose components of 'lobular' and 'ductal' may have the same cellular origin.

\section{Introduction}

Large-scale genomic analyses have demonstrated that breast cancer cells contain genetic mutations with a moderate frequency per coding megabase and high alterations of PIK3CA, ERBB2, PTEN and CDH1 (1), compared to non-small lung cell carcinoma and colorectal carcinoma. Furthermore, microarray-based gene expression analyses have indicated that breast cancers cluster into intrinsic subtypes, such as luminal, HER2, basal-like and normal breast (NB)-like subtypes, with characteristic genetic alterations being detected in each subtype $(2,3)$. For instance, the luminal A subtype is associated with frequent alterations of PIK3CA, GATA3 and FOXA1, while TP53, PIK3Ca and GATA3 are altered in the luminal B subtype, HER2 and TP53 in the HER2 subtype, and TP53, RB1, PTEN and CCNE1 in the basal-like subtype (4). Although breast cancer patients markedly benefit from personalized therapy (5-9), unlike patients with non-small lung cancer whose treatments are based on multiple genetic mutations, treatment strategies are primarily based on endocrine responsiveness and/or HER2 amplification rather than multiple gene mutations. Thus, large-scale genomic analyses of associated mutations and corresponding expression levels may provide an overview of the unique characteristics for each carcinoma type. 
The pathological classification of breast cancer is partially associated with intrinsic subtypes $(10,11)$ and the former has a much smaller role in designing treatment regimens compared to the latter. However, importantly, pathological morphology provides information regarding the phenotypic heterogeneity of tumors, as morphological characteristics represent the integrated sum of all molecules that are produced in cancer cells and are interconnected thereby. Hence, accurate interpretation of differences in pathological morphology is imperative to understanding carcinogenesis. A single tumor displays considerable diversity in morphology and characteristics, which is composed of diverse cell types harboring different mutations and gene expression profiles $(12,13)$. The cancer stem cell theory (14) and stochastic theory (clonal selection and expansion theory) (15) have been proposed to explain how tumors acquire such diversity. A comprehensive understanding of tumor diversity is important for deciphering the clinical symptoms and biological mechanisms underlying the sensitivity and/or resistance of primary tumors to therapeutic interventions and for effectively managing recurrence and/or metastasis.

DNA extracted from formalin-fixed paraffin-embedded (FFPE) samples has been used to reveal associations between morphology and mutational diversity in human solid cancers. Heterogeneous glucose-6-phosphate dehydrogenase (16) on the X chromosome based on the lyonization theory (17), androgen receptor polymorphism (18), loss of heterozygosity (LOH) $(19,20)$, comparative genomic hybridization (21) and oncogene analysis have been utilized to analyze the clonality of breast cancer subtypes. However, these methods are insufficient to determine the lineage of all tumor cells in a patient. For instance, analysis of polymorphisms in the gene encoding the androgen receptor is limited to males, while the $\mathrm{LOH}$ method is limited to tumors induced by genetic abnormalities. Mutations accumulate in intracellular mitochondrial DNA (mtDNA) during cellular aging and tumorigenesis, particularly in the D-loop region, due to the absence of mtDNA-specific DNA repair enzymes. A previous study by our group indicated that analyzing mutations in the D-loop region is a useful technique for understanding cancer cell clonality (22). Prior to the advent of next-generation sequencing, mtDNA D-loop analysis was used to determine tumor cell clonality independent of gender or genetic abnormalities.

The objective of the present study was to elucidate the clonal relationships of cancer cells occurring in tumors exhibiting both ductal and lobular phenotypes based on morphological features using next-generation sequencing of a novel CCP, in addition to the conventional mtDNA D-loop analysis method.

\section{Materials and methods}

FFPE samples from patients with breast cancer. The samples of 464 patients with breast cancer who underwent mastectomy between January 2013 and December 2014 at Itabashi Hospital, Nihon University School of Medicine (Tokyo, Japan) were obtained. Among these, 433 had not undergone preoperative chemotherapy and 14 had been diagnosed with combined lobular and ductal carcinoma (CLDC). All tumor samples underwent routine FFPE at the pathological diagnosis departments of our hospital. The pathological diagnoses were made according to the classification of breast tumors issued by the World Health Organization (WHO) $(23,24)$. Tumors comprising $10-90 \%$ of special subtypes were defined as 'mixed invasive breast carcinoma of no special type (IBC-NST) and special subtypes' by the WHO in general. However, no distinct subtype or designation has been proposed for mixed IBC-NST and invasive lobular carcinoma. Such tumors have been given several designations, e.g., invasive ductulolobular carcinoma, invasive ductal carcinoma with lobular features, or ductal or lobular carcinoma (25). In the present study, the definition of mixed ductal-lobular carcinoma (MDL) given by McCart Reed et al (25) was used for carcinomas in which the ductal component constitutes at least $10 \%$ of the tumor and the lobular component constitutes $\geq 50 \%$. It is also possible that multiple ductal and lobular lesions are present in the same breast. Therefore, the term, CLDC was used, which was defined by Tazaki et al (26) for breast carcinoma wherein lobular carcinoma coexists with ductal carcinoma on the same side of the breast. Of the 14 CLDC cases, two with tumors comprising lesions that contained sufficient DNA for analytical purposes (1 $\mu \mathrm{g}$ or more) following laser microdissection (LMD), were selected for the present study.

Case 1 comprised a main tumor in the outer cranial region, an MDL lesion consisting of invasive lobular carcinoma (ILC), lobular carcinoma in situ (LCIS), invasive ductal carcinoma (IDC) and ductal carcinoma in situ (DCIS1) (Fig. 1 and Tables I and II). An additional DCIS2 was also present in the outer caudal region. DCIS1 and DCIS2 were discontinuous and separate lesions. Flat epithelial atypia (FEA) was spread throughout the mammary gland. Skin and lymph nodes, which are extramammary non-neoplastic tissues (EMNT), were used as the control.

The samples from case 2 included IDC, ILC, LCIS and FEA. These lesions were located in close proximity to each other (Tables I and II). EMNT was obtained from lymph nodes.

A total of 8 samples were obtained for case 1 and five samples for case 2 . Case 1 was a 46 -year-old female, and case 2 was a 41-year-old female. Features of the patients, the samples and experiments are listed in Tables I and II.

The present study was performed in accordance with the stipulations of the Declaration of Helsinki and was approved by the institutional review board of the Nihon University School of Medicine Ethics Committee (approval nos. 147, 115 and 250-1). Written informed consent was obtained from all patients. The study was performed according to the Japanese national guidelines 'Ethical Guidelines for Medical and Health Research Involving Human Subjects' (the Ministry of Health, Labor and Welfare; Ministry of Education, Culture, Sports, Science and Technology; and Ministry of Economy, Trade and Industry).

LMD, DNA extraction and quality assessment of DNA. FFPE tissue sections $(10 \mu \mathrm{m})$ were prepared using a DIRECTOR slide (cat. no. 11505158; Leica Microsystems GmbH). Following deparaffinization, the sections were subjected to LMD using an LMD 6000 (Leica Microsystems) according to the protocol provided by the manufacturer. DNA was extracted from FFPE samples using a RecoverAll Total Nucleic Acid Isolation kit (Thermo Fisher Scientific, Inc.) with certain modifications, as described previously (27). DNA quality was determined by performing quantitative PCR (qPCR) for GAPDH using a 
Table I. Basic data in cases of combined lobular and ductal carcinoma.

\begin{tabular}{lcc}
\hline Variable & Case 1 & Case 2 \\
\hline Age, years & 46 & 41 \\
Sex & Female & Female \\
Breast side & Right & Left \\
Operation & Bp+Bt & Bp \\
Number of samples & 10 & 5 \\
\hline
\end{tabular}

Bp, breast partial resection; Bt, total mastectomy

TaqMan ${ }^{\circledR}$ Gene Expression Assay kit (cat. no. Hs02758991_g1; Thermo Fisher Scientific, Inc.). As a quality check control, the ratio of sample DNA to frozen tissue DNA was calculated using the delta quantification cycle $(\Delta \mathrm{Cq})$ method and the results are expressed as $2^{-\Delta \Delta \mathrm{Cq}}$, as described previously (27-29).

mtDNA D-loop analysis. mtDNA polymorphisms are frequently observed in mutational hot spots, designated as hypervariable regions (HV) (30). A total of two HVs were located between bases 16,024-16,383 and 57-372 (30). For PCR, six sets of primers were designed, including forward (F1-F6) and reverse sequences (R1-R6) (Table III). PCR was performed on each sample with eight different primer combinations as follows: F1-R1, F2-R2, F3-R3, F1-R4, F4-R1, F3-R5, F5-R6 and F6-R3. The reaction was performed in 20 $\mu 1$ reaction buffer of Takara Ex Taq Hot Start Version (Takara Bio, Inc.), containing $1 \mathrm{ng}$ of sample DNA, under the following conditions: Preheating at $94^{\circ} \mathrm{C}$ for $60 \mathrm{sec} ; 35$ cycles of $98^{\circ} \mathrm{C}$ for $10 \mathrm{sec}$ and $60^{\circ} \mathrm{C}$ for $30 \mathrm{sec}$ for annealing/elongation, and a final extension at $72^{\circ} \mathrm{C}$ for $5 \mathrm{~min}$. Subsequently, $10 \mu \mathrm{l}$ of the reaction products were subjected to $2 \%$ agarose gel electrophoresis with GelRed (Biotium, Inc.). Sanger sequencing was performed as described below using the reaction products eluted from electrophoretic bands.

In the absence of a consensus regarding the designation of genetic alterations in mtDNA, definitions in the present study were as follows: A single nucleotide polymorphism (SNP) was defined as a single base difference between individuals, as reported in the National Center for Biotechnology Information (NCBI) reference sequence (https://www.ncbi. nlm.nih.gov/nuccore/NC_012920), where all tissues in each case were identical. A single nucleotide variation (SNV) was defined as a single nucleotide sequence mismatch in a specific harvested tissue, differentiated from SNP. A simple sequence repeat number variation (SSRNV) was defined as a variation of the number of one or several simple base repeat sequences, in a manner that is inconsistent with repetition in EMNT. It was confirmed that the number of SSRNV repeats was not a technical artifact caused by PCR previously (31).

SSRNVs were detected in HV (Mt57-372) and in non-HV (Mt516-525). Regarding SSRNVs of mtDNA HV (Mt303-310), altered repeat numbers were observed, with the coexistence of repeat numbers C8 and C7 in NB and C8 homologically observed in neoplastic lesions. This was designated as loss of variation (LOV). Regarding SSRNVs in non-HV (516-525),
A
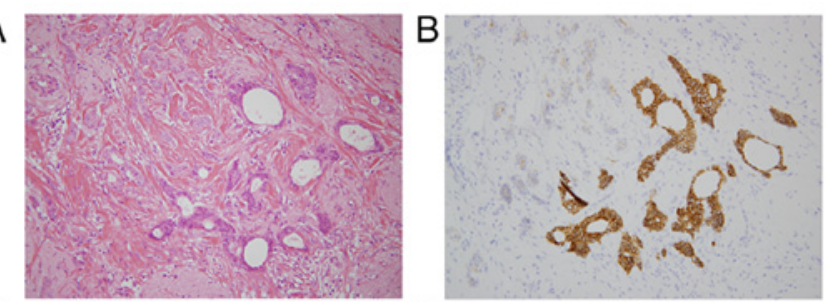

C
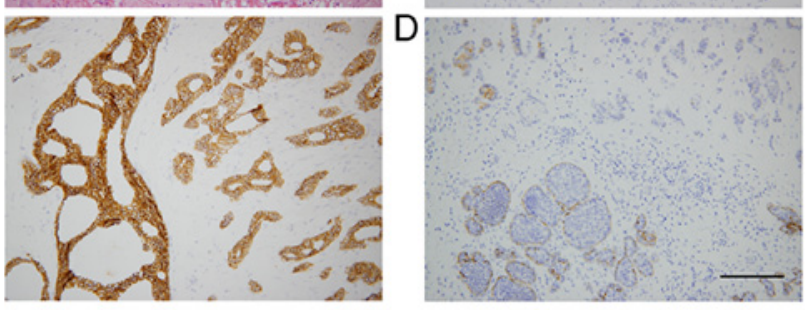

Figure 1. Histopathological images of combined ductal and lobular carcinoma (Case 1). (A) HE-staining of a region where ILC and IDC are adjacent to each other. The ILC is on the left side of the image and the IDC is on the right side. (B) Immunohistochemical staining for E-cadherin in the region identical to the HE-stained region. IDC is positive for E-cadherin at the membrane and negative for ILC. (C) Immunohistochemical staining for E-cadherin in ductal carcinoma. DCIS is present on the left side of the image and IDC is present on the right side of the image. Both types are E-cadherin membrane-positive. (D) Immunohistochemical staining for E-cadherin in lobular carcinoma. LCIS is present on the left and ILC is present on the right. Both cancers are negative for E-cadherin (scale bar, $200 \mu \mathrm{m}$ ). DCIS, ductal carcinoma in situ; IDC, invasive ductal carcinoma; ILC, invasive lobular carcinoma; LCIS, lobular carcinoma in situ.

the homological repeat of CA7 in normal tissue with acquired coexistence of CA6/7 in neoplastic lesions was observed, for which it was designated as a gain of variation (GOV).

Amplicon sequencing. DNA from 9 samples (case 1: EMNT, DCIS2, IDC and ILC; case 2: EMNT, NB, FEA, IDC and LCIS) (Tables I and II), were used for amplicon sequencing by an Ion AmpliSeq Comprehensive Cancer Panel (CCP) on an Ion Torrent platform (Thermo Fisher Scientific, Inc.) according to the manufacturers' protocols with specific modifications as below. Although the manufacturer's protocol recommended to check the amount of DNA using Qbit, the current study checked it according to the method reported by Nakayama et al (27). The panel consists of $\sim 16,000$ primer pairs covering all exons of 409 cancer-associated genes (1.6 megabases of target sequences). DNA (120 ng) was used to prepare the libraries with IonXpress barcoded adapters. An Ion Proton Sequencer (Thermo Fisher Scientific, Inc.) was used for sequencing with the ion chip.

Sequencing results were analyzed using the Torrent Suite Software (Thermo Fisher Scientific, Inc.). Candidates for cancer-specific SNVs were selected by Tumor-Normal pair analysis version 4.2 of Ion Reporter (Thermo Fisher Scientific, Inc.) using the following filters: $>100$ total reads; $>10 \%$ of mutation rate; and 0 reads of variant in a control non-mammary and non-tumor sample. Interpretation of the clinical significances of detected gene mutations was conducted by referring the oncology database of Precision Oncology Knowledge Base (OncoKB, https://www.oncokb.org/) and Catalog of Somatic Mutations In Cancer (COSMIC, https://cancer.sanger.ac.uk/cosmic).

SYBR green allele-specific qPCR. SNVs detected by amplicon sequencing were validated using SYBR-Green allele-specific 
Table II. Analysis of data in cases of combined lobular and ductal carcinoma.

\begin{tabular}{|c|c|c|c|c|c|c|c|c|}
\hline \multirow[b]{2}{*}{ Histological type } & \multicolumn{4}{|c|}{ Case 1} & \multicolumn{4}{|c|}{ Case 2} \\
\hline & mtDNA & dPCR & $\mathrm{CCP}$ & MDA & mtDNA & dPCR & $\mathrm{CCP}$ & MDA \\
\hline Extramammary non-neoplastic tissue & + & + & + & + & + & + & + & + \\
\hline Normal breast & + & + & / & + & + & / & + & + \\
\hline Flat epithelial atypia & + & + & l & + & + & + & + & + \\
\hline Ductal carcinoma in situ 1 & + & + & l & + & - & - & - & - \\
\hline Ductal carcinoma in situ 2 & + & + & + & + & - & - & - & - \\
\hline Invasive ductal carcinoma & + & + & + & + & + & + & + & + \\
\hline Lobular carcinoma in situ & + & + & l & + & + & + & + & + \\
\hline Invasive lobular carcinoma & + & l & + & + & - & - & - & - \\
\hline
\end{tabular}

+, Examined; /, not available; -, no tissue submitted for pathological examination; mtDNA, mitochondrial DNA; CCP, comprehensive cancer panel; MDA, mutation detection assay; dPCR, digital PCR.

Table III. Primers for direct sequencing of mtDNA D-loop region.

\begin{tabular}{lll}
\hline Primer name & \multicolumn{1}{c}{ NCBI no. } & Primer sequence \\
\hline F1 & Mt16065-16084 & 5'-TGACTCACCCATCAACAACC-3' \\
F2 & Mt16422-16441 & 5'-AATATCCCGCACAAGAGTGC-3' \\
F3 & Mt111-130 & 5'-ACCCTATGTCGCAGTATCTG-3' \\
F4 & Mt16196-16205 & 5'-ATGCTTACAAGCAAGTACAG-3' \\
F5 & Mt311-330 & 5'-TCCCCCCGCTTCTGGCCACA-3' \\
F6 & Mt528-547 & 5'-GCTGCTAACCCCATACCCCG-3' \\
R1 & Mt16491-16510 & 5'-GGAACCAGATGTCGGATACA-3' \\
R2 & Mt244-263 & 5'-CGGCTGTGCAGACATTCAAT-3' \\
R3 & Mt585-604 & 5'-GCTTTGAGGAGGTAAGCTAC-3' \\
R4 & Mt16153-16172 & 5'-TTGGGTTTTTATGTACTACA-3' \\
R5 & Mt283-302 & 5'-TTTGGTGGAAATTTTTTGTT-3' \\
R6 & Mt495-514 & 5'-ACCCCCGCCCATCCTACCCA-3' \\
\hline
\end{tabular}

MtDNA, mitochondrial DNA; NCBI, National Center for Biotechnology Information.

qPCR. A number of genetic mutations were detected in case 2; therefore, the highest and second-highest mutation rates were selected for validation analysis, resulting in four genes in case 2 being selected. The target genes of interest, which were validated with SYBR-Green allele-specific qPCR, are presented in Table SI. The small sample size in case 2 was accounted for by pre-amplifying the region targeted for verification using specific primer sets (Table SII). A total of $10 \mathrm{ng}$ of sample DNA were allowed to react with $20 \mu \mathrm{l}$ of Takara Ex Taq Hot Start Version (Takara Bio, Inc.) in the presence of two sets of case 2 primers (KMT2C and CDH1) as follows: Preheating at $94^{\circ} \mathrm{C}$ for $60 \mathrm{sec} ; 45$ cycles of $98^{\circ} \mathrm{C}$ for $10 \mathrm{sec}$ and $60^{\circ} \mathrm{C}$ for $30 \mathrm{sec}$ as the annealing temperature; and a final extension at $72^{\circ} \mathrm{C}$ for $5 \mathrm{~min}$. PCR was performed with two sets of case 2 primers (PIK3CA and TIMP3) as follows: Preheating at $94^{\circ} \mathrm{C}$ for $60 \mathrm{sec} ; 45$ cycles of $98^{\circ} \mathrm{C}$ for $10 \mathrm{sec}$ and $55^{\circ} \mathrm{C}$ for $30 \mathrm{sec}$ as the annealing temperature; $72^{\circ} \mathrm{C}$ for $60 \mathrm{sec}$; and a final extension at $72^{\circ} \mathrm{C}$ for $5 \mathrm{~min}$.
Allele-specific qPCR was performed using the Thunderbird SYBR qPCR Mix (Toyobo Life Science) with StepOnePlus (Thermo Fisher Scientific, Inc.) and two primer sets. Allele-specific primers for wild-type and mutant sequences, as well as single reversely-directed primers, were designed by rectifying melting temperatures to a similar temperature, based on the Nearest Neighbor's method for melting temperature calculation (32). PCR was performed in a $10-\mu 1$ reaction by preheating at $95^{\circ} \mathrm{C}$ for $60 \mathrm{sec}$, followed by 40 cycles of $95^{\circ} \mathrm{C}$ for $15 \mathrm{sec}$ and 60 to $65^{\circ} \mathrm{C}$ for $60 \mathrm{sec}$. Annealing temperatures for each primer set are presented in Table SI. The ratio of mutant allele to wild-type allele was calculated as $2^{-\Delta \Delta \mathrm{Cq}}$, in which $\Delta \mathrm{Cq}$ is calculated by subtracting the $\mathrm{Cq}$ value of wild-type PCR from that of mutant PCR. The mutant allele frequency

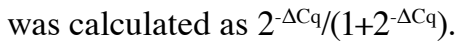

The primer sets for the wild-type region of genes with mutations were prepared and compared based on a quantitative curve using SYBR green as the fluorescent probe. 


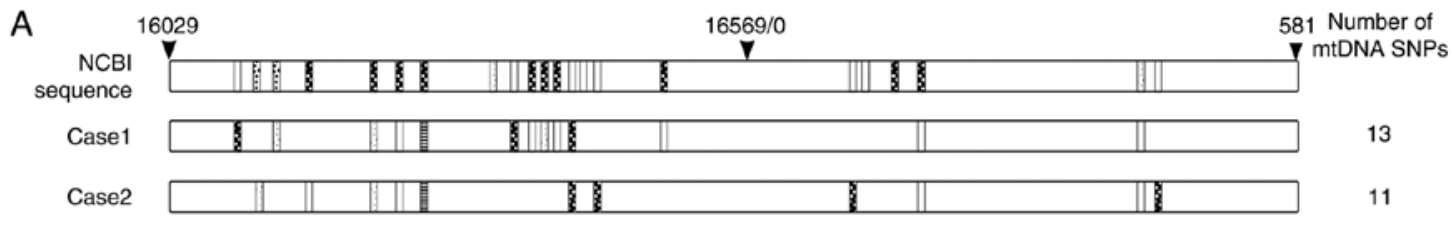

SNP: single nucleotide polymorphysm 0 ,

T A G C Deletion

B

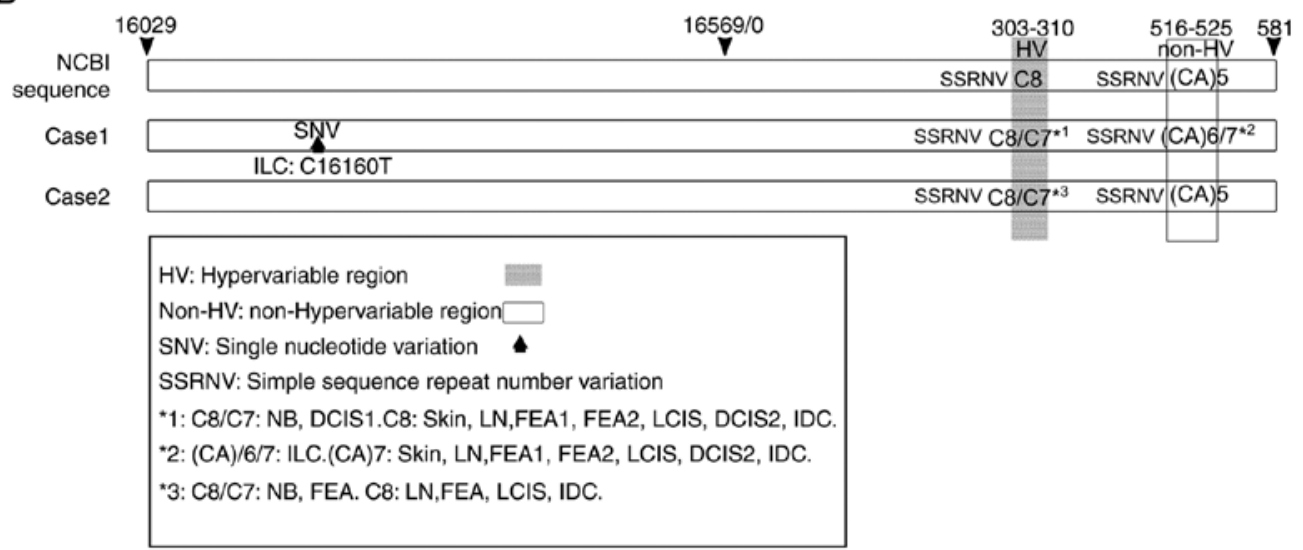

Figure 2. SNPs, SNVs and SSRNVs detected in the mtDNA D-loop region. White rectangles indicate the entire region of the mtDNA D-loop. Sequence data from NCBI are provided as a reference. (A) SNPs detected in non-tumor tissue of cases 1 and 2 are presented. (A) Small rectangular blocks indicate mitochondrial SNPs and the density of dots in each block corresponds to each base (T, A, G and C). (B) SNVs and SSRNVs detected in non-tumor tissue of cases 1 and 2. Shaded areas indicate HV regions and white square areas indicate non-HV regions; upward solid black arrows indicate SNVs. "1, C8/C7 detected in NB and DCIS1; C8 detected in skin, LN, FEA1, FEA2, LCIS, DCIS2 and IDC. "2, (CA)6/7 detected in ILC; (CA)7 detected in skin, LN, NB, FEA1, FEA2, LCIS, DCIS1, DCIS2 and IDC. "3, C8/C7 detected in NB and FEA; C8 detected in LN, FEA, LCIS and IDC. FEA, flat epithelial atypia; NB, normal breast; DCIS, ductal carcinoma in situ; IDC, invasive ductal carcinoma; ILC, invasive lobular carcinoma; LCIS, lobular carcinoma in situ; LN, lymph node; NCBI, National Center for Biotechnology Information; SNV, single nucleotide variant; SSRNV, simple sequence repeat number variation; SNP, single nucleotide polymorphism; $\mathrm{HV}$, hypervariable region; mtDNA, mitochondrial DNA; CA, Cytosine and adenine.

Mutation analysis was performed based on the differences in amplification efficiency. The primer sequences are presented in Table SI. The results were verified via SYBR Premix Ex Taq (Takara Bio, Inc.) according to the manufacturer's protocol. The mutant allele frequency was calculated as described in the previous paragraph.

Sanger sequencing for mtDNA D-loop. PCR products of the amplified mtDNA D-loop region were purified using an Illustra ExoProStar 1-Step kit (Cytiva) and sequenced using the BigDye Terminator version 1.1 cycle sequencing kit (Thermo Fisher Scientific, Inc.). Following purification using the BigDye Xterminator kit (Thermo Fisher Scientific, Inc.), nucleotide sequences were determined using an ABI310 Genetic Analyzer (Thermo Fisher Scientific, Inc.).

Sanger sequencing for insertion/deletion mutation detected by amplicon sequencing. A total of two insertion/deletion mutations, namely $\mathrm{CDH} 1$ (T>TA at chr16:68842455) of case 1 and KMT2C (AG>A at chr7:151860111) of case 2, detected by amplicon sequencing, were examined via Sanger sequencing. PCR and Sanger sequencing of CDH1 and KMT2C were performed using the same method that was described for mtDNA D-loop using the primer pairs presented in Table SII.

Digital PCR. CDH1 and VPS45A were selected for verification of the loss of chromosome 16q and gain of chromosome 1q, respectively. RNase $P$, a reference assay probe, was used as an endogenous control. The Quant Studio ${ }^{\mathrm{TM}}$ 3D Digital PCR System (Thermo Fisher Scientific, Inc.) was used for qPCR. DNA samples $(60 \mathrm{ng})$ were used for the copy number variation (CNV) assay along with the TaqMan ${ }^{\circledR}$ Copy Number Assay probe reagent for CDH1 (assay ID, Hs05461677_cn; assay location, chr16:68800293; amplicon length, $88 \mathrm{bp}$ ) or VPS45A (assay ID, Hs02288810_cn; Thermo Fisher Scientific, Inc.; assay location, chr1:150117372; amplicon length, 86 bp), TaqMan ${ }^{\circledast}$ Copy Number Reference Assay probe for RNaseP [positon, chr14q11.2(20811565) on NCBI build 37; Thermo Fisher Scientific, Inc.] and QuantStudio ${ }^{\mathrm{TM}}$ 3D Digital PCR Master Mix v.2 (Thermo Fisher Scientific, Inc.) according to the manufacturer's instructions. Distilled water was added to adjust the total volume to $15 \mu \mathrm{l}$. The negative control reaction, which was performed in a total volume of $16 \mu$ l, contained no DNA. This negative control sample was used for each round of thermocycling.

For each sample, $15 \mu \mathrm{l}$ of each prepared reaction mixture was loaded onto a QuantStudio 3D ${ }^{\mathrm{TM}}$ Digital PCR 20K Chip Kit v.2 (Thermo Fisher Scientific, Inc.) using an automatic chip loader and the signal was amplified using the following thermocycling conditions: $95^{\circ} \mathrm{C}$ for $8 \mathrm{~min} ; 45$ cycles at $95^{\circ} \mathrm{C}$ for $15 \mathrm{sec}$ and $56^{\circ} \mathrm{C}$ for $1 \mathrm{~min}$; and a final extension step at $60^{\circ} \mathrm{C}$ for $2 \mathrm{~min}$. Amplification followed by chip imaging and secondary analysis was performed using the QuantStudio 3D Analysis Suit Software (version 3.0.2.2; Thermo Fisher Scientific, Inc.). 
The results of the analysis yielded the copy numbers of target genes (CDH1 and VPS45A) and a reference gene (RNAseP) per microliter of each sample, which were used to calculate the ratio of target genes to a reference gene. These calculations were based on the theory that loss of chromosome $16 \mathrm{q}$ and gain of $1 \mathrm{q}$ resulted in haploid and triploid copy numbers per cell, respectively. The copy number of the target genes (copies/ $\mu \mathrm{l}$ ) was divided by that of the reference gene (copies $/ \mu \mathrm{l})$ and the obtained ratios of $\mathrm{CDH} 1$ or VPS45A to RNAseP in each sample were corrected, so that the ratios of CDH1 or VPS45A to RNAseP of EMNT were 1. Usually, a normal cell has two copies of CDH1 and RNAseP. A single 16q-loss cell has only one copy of CDH1 and two copies of RNAseP. When the total sum of 16q-loss cells and normal cells is $100 \%$, the proportion of $16 q$-loss cells may be calculated by solving the two sets of simultaneous equations. The same calculations were performed as for 1q gain cells with normal cells having two copies of VPS45A and two copies of RNAseP, while the 1q triploid cells had three copies of VPS45A and two copies of RNAseP.

\section{Results}

Cancer-specific alterations in the mtDNA D-loop. The results indicated that mtDNA SNPs were present at 13 and 11 sites in the D-loops of cases 1 and 2, respectively (Fig. 2A). SNPs were detected by referring to the NCBI reference database. Only one SNV was detected at the 16,160 locus in ILC samples (case 1; Table IV, Fig. 2B).

SSRNVs were detected in two regions, one of which was in HV (base numbers 303-310), while the other one was in non-HV (base numbers 516-525; Fig. 2B). For SSRNVs in HV, the coexistence of $\mathrm{C} 8$ and $\mathrm{C} 7$ was detected in NB and DCIS1 (case 1) and NB and FEA (case 2) (Table IV, Fig. 2B). For SSRNVs in non-HV, the coexistence of CA7 and CA6 was detected in ILC (case 1) (Table IV, Fig. 2B).

Loss of chromosome $16 q$ and gain of $1 q$. In case 1, the estimated proportions of cells with a loss of chromosome $16 \mathrm{q}$ were as follows: FEA, 37.2\%; DCIS1, 0\%; DCIS2, 65.0\%; IDC, 36.8\%; LCIS, 46.2\%; and ILC, $47.8 \%$ (Tables V and VI). The estimated proportion of cells with a 1q gain was as follows: FEA, 35.0\%; DCIS1, 31.0\%; DCIS2, 131.4\%; IDC, 82.6\%; LCIS, 97.8\%; and ILC, $75.0 \%$ (Tables V and VI).

In case 2 , the estimated proportions of cells with a loss of chromosome 16q were as follows: FEA, 0\%; LCIS, 31.2\%; and IDC, $12.8 \%$ (Tables $\mathrm{V}$ and VI). The estimated proportions of cells with a gain of 1q were: FEA, 59.4\%; LCIS, 39.6\%; and IDC, $91.8 \%$ (Tables V and VI).

Cancer-specific variations in 409 cancer-associated genes. The number of mutations that satisfied adopted filters (described in the Materials and methods) was 5 and 15 in cases 1 and 2, respectively (Table SIII). Data for CCP, gene locus, altered genotype and variant frequency calculated by dividing variant allele coverage by total coverage, are presented in Table SIII. The mutation distribution among different lesions in each case is represented by a Venn diagram (Fig. 3). In case 1, DCIS2, IDC and ILC carried three, one and two mutations, respectively. IDC and ILC shared one mutation, while DCIS2 carried 3 independent mutations. DCIS1 did not share any common
Table IV. Results of the mitochondrial DNA D-loop region analysis.

\begin{tabular}{lcccc}
\hline Item & Case 1 & & Case 2 \\
\hline Type of variation & SNV & SSRNV & SSRNV & SSRNV \\
NCBI no. & 16160 & $303-310$ & $516-525$ & $303-310$ \\
Variation sequence & $\mathrm{C}$ & $\mathrm{C} 8$ & $(\mathrm{CA}) 7$ & $\mathrm{C} 8$ \\
EMNT & $\mathrm{C}$ & $\mathrm{C} 8$ & $(\mathrm{CA}) 7$ & $\mathrm{C} 8$ \\
NB & $\mathrm{C}$ & $\mathrm{C} 8 / \mathrm{C} 7$ & $(\mathrm{CA}) 7$ & $\mathrm{C} 8 / \mathrm{C} 7$ \\
FEA & $\mathrm{C}$ & $\mathrm{C} 8$ & $(\mathrm{CA}) 7$ & $\mathrm{C} 8 / \mathrm{C} 7$ \\
DCIS1 & $\mathrm{C}$ & $\mathrm{C} 8 / \mathrm{C} 7$ & $(\mathrm{CA}) 7$ & - \\
DCIS2 & $\mathrm{C}$ & $\mathrm{C} 8$ & $(\mathrm{CA}) 7$ & - \\
IDC & $\mathrm{C}$ & $\mathrm{C} 8$ & $(\mathrm{CA}) 7$ & $\mathrm{C} 8$ \\
LCIS & $\mathrm{C}$ & $\mathrm{C} 8$ & $(\mathrm{CA}) 7$ & $\mathrm{C} 8$ \\
ILC & $\mathrm{C} / \mathrm{T}$ & $\mathrm{C} 8$ & $(\mathrm{CA}) 7 /(\mathrm{CA}) 6$ & - \\
\hline
\end{tabular}

-, no tissue submitted for pathological examination; NCBI, National Center for Biotechnology Information; SNV, single nucleotide variant; SSRNV, simple sequence repeat number variation; EMNT, extramammary non-neoplastic tissue; FEA, flat epithelial atypia; $\mathrm{NB}$, normal breast; DCIS, ductal carcinoma in situ; IDC, invasive ductal carcinoma; ILC, invasive lobular carcinoma; LCIS, lobular carcinoma in situ. $\mathrm{C}$, cytosine; $\mathrm{T}$, thymine; CA, cytosine and adenine.

mutations with other lesions, including DCIS2. In case 2, FEA, IDC and LCIS carried four, six and 10 mutations, respectively. FEA and IDC shared three, IDC and LCIS shared two, and FEA and LCIS shared one mutation. Furthermore, one common mutation was shared by FEA, IDC and LCIS, and FEA (Fig. 3).

Variant frequencies of gene mutations detected by the CCP are provided in Table SIII and the genes submitted for verification by allele-specific PCR are presented in Table VII. For each sample, the results of allele-specific PCR are presented in Table VIII. In case 1, the calculated mutation rate of $\mathrm{CDH} 1$ was $15 \%$ in IDC, $30 \%$ in LCIS and $34 \%$ in ILC. CDH1 mutation (p.Asn174fs; likely oncogenic according to OncoKB, no entry in COSMIC) was detected in IDC and ILC samples. However, it was not observed in any of the other samples, indicating that the same mutation was shared by IDC and ILC. Mutations in RRM1 existed only in ILC, while mutations of AKT1, RALGDS and PIK3CA existed only in DCIS2, confirming the results obtained with the CCP. In addition, CDH1 mutations (T>TA at chr16:68842455) were detected using Sanger sequencing (Fig. 4).

In case 2, a nonsense mutation of CDH1 (p.Gln23Ter, likely oncogenic according to OncoKB, frequent 70 mutations reported in COSMIC) (Fig. 4) existed only in LCIS. Mutation of TIMP3 was shared by FEA and IDC. Mutation of PIK3CA was shared in NB, FEA and IDC; these results were consistent with those from the CCP. Mutations in KMT2C were not detected by SYBR green allele-specific qPCR, supporting the result of the $\mathrm{CCP}$, which indicated a deletion in KMT2C in IDC samples of case 2 (Fig. 4).

Summary of mtDNA mutation analysis and verification of results of $C N V$ and $C C P$ by $q P C R$. A schematic of the progression of mutations in the carcinoma cells of different 
Table V. Results of digital PCR with regard to $16 \mathrm{q}$ loss

\begin{tabular}{|c|c|c|c|c|c|}
\hline Case/tissue type & CDH1 (copies/ $\mu \mathrm{l}$ ) & RNaseP (copies/ $\mu \mathrm{l})$ & CDH1/RNaseP & Corrected value $^{\mathrm{a}}$ & Loss cells ${ }^{\mathrm{b}}(\%)$ \\
\hline \multicolumn{6}{|l|}{ Case 1} \\
\hline EMNT & 572.84 & 564.29 & 1.02 & 1.00 & 0.0 \\
\hline FEA & 286.06 & 342.03 & 0.84 & 0.82 & 37.2 \\
\hline DCIS 1 & 533.63 & 502.71 & 1.06 & 1.05 & 0.0 \\
\hline DCIS2 & 266.08 & 388.53 & 0.69 & 0.68 & 65.0 \\
\hline IDC & 281.67 & 340.02 & 0.83 & 0.82 & 36.8 \\
\hline LCIS & 278.52 & 356.85 & 0.78 & 0.77 & 46.2 \\
\hline ILC & 256.61 & 331.98 & 0.77 & 0.76 & 47.8 \\
\hline \multicolumn{6}{|l|}{ Case 2} \\
\hline EMNT & 507.97 & 466.36 & 1.09 & 1.00 & 0.0 \\
\hline FEA & 403.69 & 355.53 & 1.14 & 1.05 & 0.0 \\
\hline LCIS & 444.78 & 484.36 & 0.92 & 0.84 & 31.2 \\
\hline IDC & 535.35 & 523.79 & 1.02 & 0.94 & 12.8 \\
\hline
\end{tabular}

${ }^{\mathrm{a}}$ Corrected value of Target/RNaseP relative to EMNT sample set as 1.0 ; ${ }^{\mathrm{b}}$ calculated proportion of $16 \mathrm{q}$ loss relative to $16 \mathrm{q}$ loss cells in EMNT sample set as 1.0. EMNT, extramammary non-neoplastic tissue; FEA, flat epithelial atypia; NB, normal breast; DCIS, ductal carcinoma in situ; IDC, invasive ductal carcinoma; ILC, invasive lobular carcinoma; LCIS, lobular carcinoma in situ.

Table VI. Results of digital PCR with regard to 1q gain

\begin{tabular}{|c|c|c|c|c|c|}
\hline Case/tissue type & VPS45 (copies/ $\mu 1)$ & RNaseP (copies/ $\mu \mathrm{l})$ & VPS45A/RNaseP & Corrected value $^{a}$ & Gain cells $\mathrm{s}^{\mathrm{b}}(\%)$ \\
\hline \multicolumn{6}{|l|}{ Case 1} \\
\hline EMNT & 500.36 & 526.09 & 0.95 & 1.00 & 0.0 \\
\hline FEA & 412.35 & 368.84 & 1.12 & 1.18 & 35.0 \\
\hline DCIS 1 & 512.35 & 466.55 & 1.10 & 1.16 & 31.0 \\
\hline DCIS2 & 621.01 & 394.09 & 1.58 & 1.66 & 131.4 \\
\hline IDC & 400.64 & 298.08 & 1.34 & 1.41 & 82.6 \\
\hline LCIS & 494.98 & 349.57 & 1.42 & 1.49 & 97.8 \\
\hline ILC & 459.51 & 351.33 & 1.31 & 1.38 & 75.0 \\
\hline \multicolumn{6}{|l|}{ Case 2} \\
\hline EMNT & 421.31 & 380.38 & 1.11 & 1.00 & 0.0 \\
\hline FEA & 523.07 & 363.02 & 1.44 & 1.30 & 59.4 \\
\hline LCIS & 560.53 & 421.00 & 1.33 & 1.20 & 39.6 \\
\hline IDC & 843.61 & 521.68 & 1.62 & 1.46 & 91.8 \\
\hline
\end{tabular}

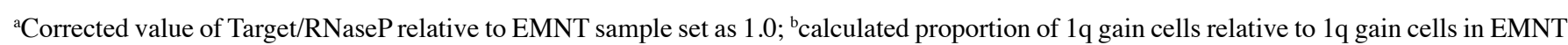
set as 1.0. EMNT, extramammary non-neoplastic tissue; FEA, flat epithelial atypia; NB, normal breast; DCIS, ductal carcinoma in situ; IDC, invasive ductal carcinoma; ILC, invasive lobular carcinoma; LCIS, lobular carcinoma in situ.

histological types in each lesion is provided in Fig. 5. In case 1, DCIS1 cancer cells harbored a 1q gain and FEA exhibited additional alterations of $16 q$ loss and mtDNA LOV; this indicated that the two pathways diverged from FEA, wherein an MDL lesion was characterized by an increase in the ratio of $16 \mathrm{q}$ loss and acquired CDH1 mutations, as detected in IDC, LCIS and ILC. ILC samples further gained an RRM1 mutation, an SNV at 16,160 and mtDNA GOV. The second pathway was associated with distinct genetic alterations (AKT1, RALGDS and PIK3CA) in DCIS2 that were topologically located in a different area from that of IDC, LCIS and ILC. These genetic alterations were not shared by DCIS1, IDC, LCIS or ILC in an MDL lesion, suggesting that the cancer cells in DCIS2 were of a different clonal origin (Fig. 5). Since FEA had a higher ratio of cells exhibiting 16q loss and 1q gain, as well as a higher homogenization of mtDNA LOV compared to those of DCIS1, FEA diagnosed under the present criteria appeared to be a neoplastic lesion similar to DCIS1.

In case 2, cancer cells in which 1q gain was detected in all lesions diverged into two paths. One branched out to LCIS with mtDNA LOV [homogenization to C8 of SSRNV in HV (303-310)], 16q loss and TIMP3 mutation. The other pathway 
Table VII. Mutation of interest detected by comprehensive cancer panel.

A, Case 1

\begin{tabular}{llllllll}
\hline Gene & \multicolumn{1}{c}{ Locus } & Type & Genotype & \multicolumn{2}{c}{ RefSeq } & Location & Function \\
\hline PIK3CA & chr3:178952085 & SNV & A/T & NM_006218.4 & Exonic & Missense & p.His1047Leu \\
RALGDS & chr9:135977481 & SNV & G/A & NM_001271775.1 & Exonic & Synonymous & p.(=) \\
RRM1 & chr11:4123150 & SNV & G/T & NM_001033.3 & Intronic & n.d. & n.d. \\
AKT1 & chr14:105246550 & INDEL & TC/TT & NM_001014431.1 & Exonic & Missense & p.Glu17Lys \\
CDH1 & chr16:68842455 & SNV & T/TA & NM_004360.3 & Exonic & Frameshift & p.Asn174fs \\
& & & & & & & insertion \\
\hline
\end{tabular}

B, Case 2

\begin{tabular}{|c|c|c|c|c|c|c|c|}
\hline Gene & Locus & Type & Genotype & RefSeq & Location & Function & Protein \\
\hline PIK3CA & chr3:178936091 & SNV & $\mathrm{G} / \mathrm{A}$ & NM_006218.2 & Exonic & Missense & p.Glu545Lys \\
\hline KMT2C & chr7:151860111 & INDEL & $\mathrm{AG} / \mathrm{A}$ & NM_170606.2 & Exonic & $\begin{array}{l}\text { Frameshift } \\
\text { deletion }\end{array}$ & p.Pro3517fs \\
\hline CDH1 & chr16:68772218 & SNV & $\mathrm{C} / \mathrm{T}$ & NM_004360.3 & Exonic & Nonsense & p.Gln23Ter \\
\hline TIMP3 & chr22:33245493 & SNV & $\mathrm{C} / \mathrm{G}$ & $\begin{array}{l}\text { NM_003490.3/ } \\
\text { NM_000362.4 }\end{array}$ & Intronic/exonic & Missense & /p.Thr59Arg \\
\hline
\end{tabular}

SNV, single nucleotide variant; INDEL, insertion and deletion; chr, chromosome; RefSeq, reference sequence; n.d., not determined; p.(=), protein has not been analyzed, but no change is expected.

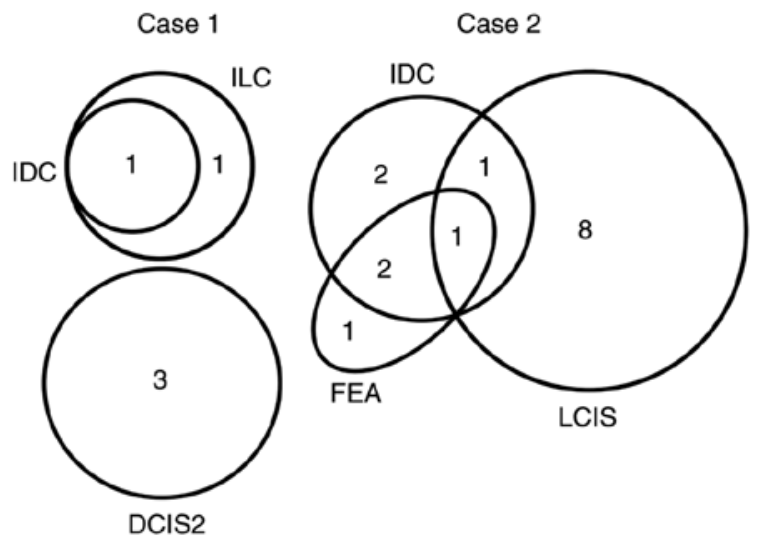

Figure 3. Venn diagrams indicating the distribution of mutations among different lesions in each case. The size of circles reflects the number of mutations within each lesion. The number of mutations is identified in the area enclosed by the circle. FEA, flat epithelial atypia; DCIS, ductal carcinoma in situ; IDC, invasive ductal carcinoma; ILC, invasive lobular carcinoma; LCIS, lobular carcinoma in situ.

involved FEA acquiring PIK3CA and TIMP3 mutations. IDC samples acquired additional KMT2C mutations, mtDNA LOV and 16q loss. In this case, LCIS, IDC and FEA were topologically similar and these lesions shared closely related mutations (Fig. 5).

\section{Discussion}

The present study demonstrated a phylogenetic association between genetic alterations in two CLDC tumors. Although only

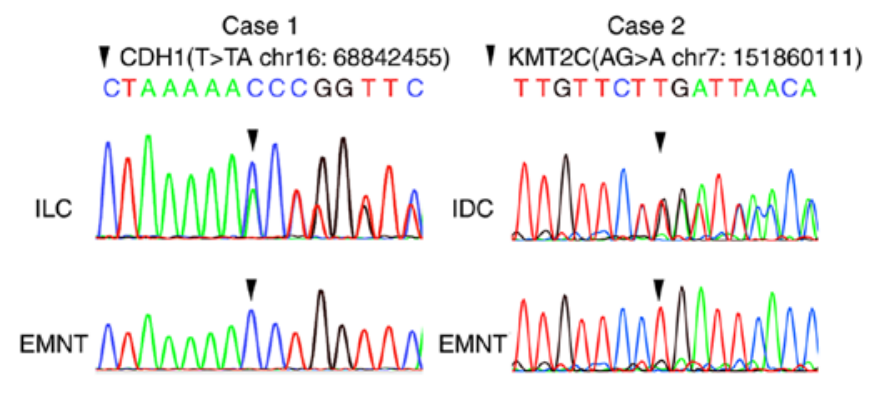

Figure 4. Sanger sequencing validation of the deletion mutation in CDH1 ( T>TA chr16:68842455) in case 1 and the insertion mutation in KMT2C (AG>A chr7:151860111) in case 2, as determined using the comprehensive cancer panel. Top panel, one representative example of the histological type in which the mutation exists in each case. At the point indicated by the downward arrowhead, a base was deleted (case 1) or inserted (case 2). As a result of insertion/deletion, the sequence after this point was shifted and duplicated waves were shown. Bottom panel, results for EMNT. Each arrowhead indicates the point of mutation. chr, chromosome; EMNT, extramammary non-neoplastic tissue; IDC, invasive ductal carcinoma; ILC, invasive lobular carcinoma.

a limited number of cases were examined, it should be emphasized that analyses performed by different methods provided consistent results for each lesion in each case. CLDC exhibited the basic gene alterations, 1q gain and/or 16q loss, and progressed to IDC or ILC as mutations accumulated. This was demonstrated using the conventional method of mtDNA mutation analysis, CNV was examined via digital PCR and the novel method of the CCP verified by SYBR green allele-specific qPCR.

A series of studies have indicated that $\mathrm{LOH}$ at chromosome $16 \mathrm{q}$ is detected in $65 \%$ of low-grade ductal carcinomas (33), 
Table VIII. Results of mutation detection assay and/or direct sequencing.

\begin{tabular}{|c|c|c|c|c|c|c|c|c|c|}
\hline \multirow[b]{2}{*}{ Histology sample type } & \multicolumn{4}{|c|}{ Case $1(\%)$} & \multicolumn{5}{|c|}{ Case $2(\%)$} \\
\hline & $\mathrm{CDH} 1$ & RRM1 & AKT1 & RALGDS & PIK3CA & PIK3CA & KMT2C & $\mathrm{CDH} 1$ & TIMP3 \\
\hline EMNT & 0 & 0 & 0 & 0 & 0 & 5 & 4 & 0 & 0 \\
\hline NB & 0 & 0 & 0 & 0 & 0 & 17 & 1 & 1 & 5 \\
\hline FEA & 0 & 0 & 0 & 0 & 0 & 31 & 0 & 0 & 40 \\
\hline DCIS1 & 0 & 0 & 0 & 0 & 0 & 1 & I & I & / \\
\hline DCIS2 & 0 & 1 & 31 & 21 & 9 & 1 & I & 1 & I \\
\hline IDC & 15 & 0 & 0 & 0 & 0 & 99 & 1 & 0 & 52 \\
\hline LCIS & 30 & 0 & 0 & 0 & 0 & 6 & 3 & 38 & 0 \\
\hline ILC & 34 & 19 & 0 & 0 & 0 & I & 1 & 1 & 1 \\
\hline
\end{tabular}

/, no sample submitted for analysis; EMNT, extramammary non-neoplastic tissue; FEA, flat epithelial atypia; NB, normal breast; DCIS, ductal carcinoma in situ; IDC, invasive ductal carcinoma; ILC, invasive lobular carcinoma; LCIS, lobular carcinoma in situ.
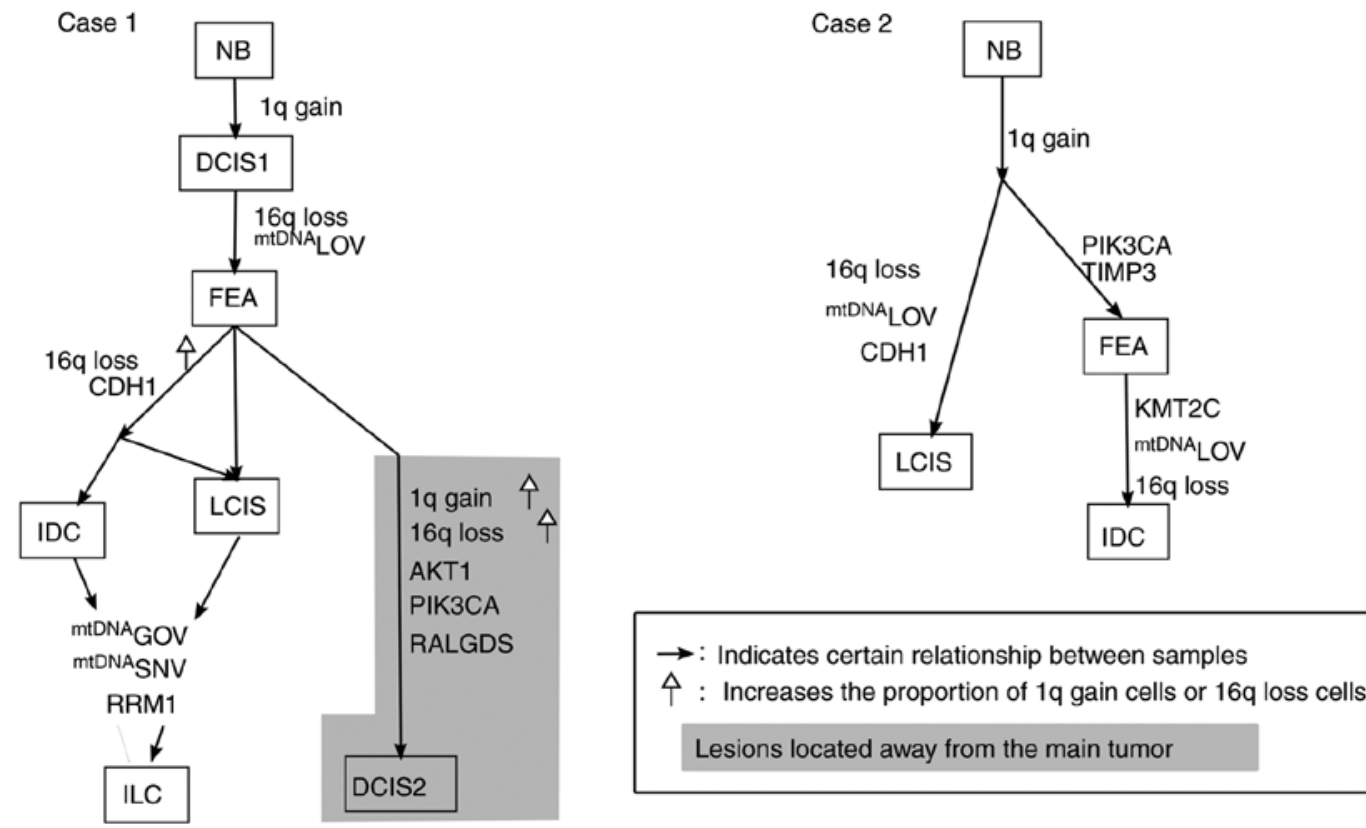

Lesions located away from the main tumor

Figure 5. Phylogenetic diagram based on the results of the present study. Solid arrows indicate correlations between lesions. Upward white arrows represent an increase in the proportion of cells with 16q loss or 1q gain. Lesions located away from the main tumor, topologically, are presented in the gray area. mtDNA, mitochondrial DNA; SNV, single nucleotide variant; FEA, flat epithelial atypia; NB, normal breast; DCIS, ductal carcinoma in situ; IDC, invasive ductal carcinoma; ILC, invasive lobular carcinoma; LCIS, lobular carcinoma in situ; LOV, loss of variation; GOV, gain of variation.

$66-78 \%$ of tubular carcinomas $(34,35)$ and $63-100 \%$ of lobular carcinomas (33-36), compared with $12 \%$ of high-grade ductal carcinomas (33). Microarray analysis indicated that the difference between low-grade (grade I and II) ductal and lobular carcinoma was $5.8 \%$, which was greater than that observed in intrinsic subtypes and histological grades. Genes exhibiting increased expression in lobular carcinomas compared with those in low-grade ductal carcinomas were associated with lipids, migration and transcription. By contrast, genes with decreased expression were related to cell adhesion (CDH1), TGF $\beta$, cytoskeleton remodeling and DNA repair ubiquitin (37). Therefore, lobular carcinomas and ductal carcinomas have been considered as distinct phenotypes with unique gene expression profiles.
However, accumulating evidence pertaining to genetic alterations indicates a close relationship between lobular neoplasia and low-grade ductal carcinoma. As loss of 16q is repeatedly reported in columnar cell lesion/FEA, lobular neoplasia, atypical ductal hyperplasia (ADH) and low-grade DCIS, and 1q gain is detected in lobular neoplasia, ADH and low-grade DCIS (38), the concept of 'low nuclear grade breast neoplasia family' was postulated (39). By contrast, high-grade DCIS lacks 1q gain or 16q loss and frequently displays $8 \mathrm{q}$ gain (38). Based on this evidence, the hypothesis that low-grade and high-grade cancers develop via different processes during the carcinogenesis of breast cancers with exceptional progression from low-grade to high-grade cancer, has been proposed (23). The results of the present 
study supported that $16 \mathrm{q}$ loss and/or 1q gain were extensively detected in such low-grade lesions.

The present study demonstrated that CLDC (excluding an MDL lesion) may undergo different genetic alterations and originate from distinct cells, even if the histological type is the same as ductal carcinoma in situ (e.g., DCIS1 and DCIS2 in case 1). With regard to MDL, the present study suggested that a CDH1 mutation was detected in IDC at a low frequency (15\%), as well as LCIS (30\%) and ILC (34\%) in the MDL lesion of case 1 . Furthermore, ILC harbored the most frequent number of genetic alterations, not only in nuclear DNA but also in the mtDNA D-loop. The present results indicated that both ductal and lobular cancer cells shared a common genetic ancestor, instead of incidentally colliding. Following a study performed using comparative genomic hybridization and whole-exome sequencing in four cases and immunohistochemical analysis of $82 \mathrm{MDLs}$, McCart Reed et al (25) reported that cancer cells with a lobular phenotype progressed from ductal components. They concluded that 'these data support a model in which separate morphological components of MDLs arise from a common ancestor and lobular morphology can arise via a ductal pathway of tumour progression'. The results of the present study support the proposed model wherein an invasive lobular carcinomatous component in mixed ductal-lobular carcinoma may arise from ductal carcinoma as well as from LCIS, but not vice versa.

Of note, a CDH1 mutation detected in an MDL lesion of case 1 (p.Agn174fs), which corresponded to the outermost domain of E-cadherin that binds to neighboring cells, was not reported in COSMIC, but detected in a mixed ductal-lobular carcinoma in a study performed at the Memorial Sloan Kettering Cancer Center (40). By contrast, CDH1 mutation in case 2 (p.Gln23Ter) is most frequently detected in lobular lesions, which is a nonsense mutation located near the $\mathrm{N}$ terminus that stops the synthesis of E-cadherin protein. Genetic alterations and subsequent transcription and/or translation result in altered protein expression, such as completely negative or incompletely positive membranous expression according to immunohistochemistry. Da Silva et al (41) reported that such aberrant E-cadherin expression may be detected in lobular carcinoma due to transcriptional repression via TGF- $\beta /$ SMAD2 activation, in addition to $\mathrm{CDH} 1$ mutation.

In the present study, tumors from cases 1 and 2 carried CDH1 mutations, in addition to deletions of 16q. The locations of assay probes used for digital PCR (location, chr16:68800293; amplicon length, 88 bp) and SYBR green allele-specific qPCR (mutation location, chr16:68842455; amplicon length, $122 \mathrm{bp}$ ) did not overlap, making it difficult to determine the exact proportion of cancer cells harboring these mutations and deletions. However, it is speculated that the following events occurred: For instance for LCIS in case 1 , the mutation was detected in $30 \%$ of $\mathrm{CDH} 1$ genes on the remnant undeleted allele, which was estimated at $77 \%$ as CDH1/RNaseP corrected by EMNT (presented as corrected value). Therefore, the percentage of $\mathrm{CDH} 1$ without mutation or deletion was $53.9 \%\{[(0.77 \times(1-0.3)] \times 100\}$ in LCIS of case 1 . In the same manner, the percentage of $\mathrm{CDH} 1$ without mutation was calculated as $50.2 \%$ in ILC of case 1 and $52.1 \%$ in LCIS of case 2, compared with that in non-tumor tissues. There are no data indicating the exact relationship between the percentage of genes without mutation or deletion, the amount of protein production and expression of CDH1 on immunohistochemistry. However, it is possible that the decrease in CDH1 without any mutation or deletion by about half (50.2-53.9\%) caused a marked decrease of E-cadherin protein synthesis, resulting in a negative reaction on immunohistochemistry. At present, it is unclear whether mutations occur on the remnant allele in the same cancer cells with deletion, or on alleles in different cells with deletion. However, it is certain that the levels of CDH1 without mutation or deletion were decreased to approximately half in total.

There were a few limitations to the present study. First, MDL and CLDL had small sample sizes. The cancer cells in an MDL lesion examined in the present study were suggested to be of the same origin, despite having different 'lobular' or 'ductal' morphological features. It has not been clarified whether the cellular origin is constant for MDL. Furthermore, limited methodologies were used for examination. mtDNA mutation analysis and CNV and CCP for nuclear DNA (verified by qPCR) were examined, while it was not clarified how the expression and methylation status altered genes. As for the examination of $\mathrm{CNV}$, analysis of the $1 \mathrm{q}$ gain and $16 \mathrm{q}$ loss were limited to the narrow region of VPS45A on 1q and CDH1 on 16q. Finally, aberrant results were obtained for E-cadherin in lobular neoplasia; the relationship between genetic alterations and these aberrant immunohistochemical findings were not clarified. These limitations will be addressed in future studies and the carcinogenesis of MDL and CLDL will be clarified.

In conclusion, the present study demonstrated that an MDL lesion is composed of cancer cells with closely related genetic characteristics. Thus, it is likely that adjacent lobular and ductal carcinomas arose from the same origin. MDL should be defined as a distinct category that is separate from CLDC, which includes multiple incidentally coexisting lesions in its definition. Although breast cancers occasionally exhibit morphological diversity, understanding the relationship between genetic alterations and morphology will help elucidate mechanisms underlying the development of cancer and aid in the development of therapeutic interventions for recurrent metastasized cancers.

\section{Acknowledgements}

The authors would like to thank Dr Hiromi Yamaguchi and Ms. Yukari Hirotani (Department of Pathology and Microbiology, Nihon University School of Medicine) for their helpful technical guidance and assistance. The authors would also like to thank Professor Keiichiro Tada (Department of Surgery, Nihon University School of Medicine), for their collaboration, clinical guidance and assistance.

\section{Funding}

This work was supported by JSPS KAKENHI (grant nos. 24590433 and 15K08357), a grant from the MEXT-Supported Program for the Strategic Research Foundation at Private Universities (grant no. S1091023) and a Nihon University Multidisciplinary Research Grant (grant no. M14-012). 


\section{Availability of data and materials}

The datasets used and/or analyzed during the current study are available from the Japanese Genotype-phenotype Archive (accession no. JGAS000300). Any further clinical data, they are available from the corresponding author upon reasonable request.

\section{Authors' contributions}

The present study plan was proposed by SM and all of the experiments were performed by HK. ME and TN significantly contributed to acquisition, analysis and interpretation of the data obtained. ME provided advice on molecular technologies, and TN confirmed that SSRNV of mtDNA was not a technical artifact caused by PCR. HK, SM and YN interpreted the data, and were involved in critically drafting and revising the results of this study. HK and SM wrote the manuscript, and all of the authors read and approved the final manuscript. HK, SM, YN and ME confirmed the authenticity of the raw data.

\section{Ethics approval and consent to participate}

The study was approved by the institutional review board of the Nihon University School of Medicine Ethics Committee (Tokyo, Japan; approval nos. 147, 115 and 250-1). Written informed consent was obtained from all patients.

\section{Patient consent for publication}

Written informed consent was obtained from all patients for publication of analysis results and registration of research data in the database

\section{Competing interests}

The authors declare that they have no competing interests.

\section{References}

1. AACR Project GENIE Consortium: AACR Project GENIE: Powering precision medicine through an international consortium. Cancer Discov 7: 818-831, 2017.

2. Perou CM, Sørlie T, Eisen MB, van de Rijn M, Jeffrey SS, Rees CA, Pollack JR, Ross DT, Johnsen H, Akslen LA, et al: Molecular portraits of human breast tumours. Nature 406: 747-752, 2000

3. Sørlie T, Perou CM, Tibshirani R, Aas T, Geisler S, Johnsen H, Hastie T, Eisen MB, van de Rijn M, Jeffrey SS, et al: Gene expression patterns of breast carcinomas distinguish tumor subclasses with clinical implications. Proc Natl Acad Sci USA 98: 10869-10874, 2001

4. Cancer Genome Atlas Network: Comprehensive molecular portraits of human breast tumours. Nature 490: 61-70, 2012.

5. Waks AG and Winer EP: Breast cancer treatment: A Review. JAMA 321: 288-300, 2019.

6. Early Breast Cancer Trialists' Collaborative Group (EBCTCG); Davies C, Godwin J, Gray R, Clarke M, Cutter D, Darby S, McGale P, Pan HC, Taylor C, et al: Relevance of breast cancer hormone receptors and other factors to the efficacy of adjuvant tamoxifen: Patient-level meta-analysis of randomised trials. Lancet 378: 771-784, 2011.

7. Slamon DJ, Clark GM, Wong SG, Levin WJ, Ullrich A and McGuire WL: Human breast cancer: Correlation of relapse and survival with amplification of the HER-2/neu oncogene. Science 235: 177-182, 1987.
8. Cobleigh MA, Vogel CL, Tripathy D, Robert NJ, Scholl S, Fehrenbacher L, Wolter JM, Paton V, Shak S, Lieberman G and Slamon DJ: Multinational study of the efficacy and safety of humanized anti-HER2 monoclonal antibody in women who have HER2-overexpressing metastatic breast cancer that has progressed after chemotherapy for metastatic disease. J Clin Oncol 17: 2639-2648, 1999.

9. Burstein HJ, Curigliano G, Loibl S, Dubsky P, Gnant M, Poortmans P, Colleoni M, Denkert C, Piccart-Gebhart M, Regan M, et al: Estimating the benefits of therapy for early-stage breast cancer: The St. Gallen international consensus guidelines for the primary therapy of early breast cancer 2019. Ann Oncol 30: 1541-1557, 2019.

10. Weigelt B, Geyer FC and Reis-Filho JS: Histological types of breast cancer: How special are they? Mol Oncol 4: 192-208, 2010.

11. Weigelt B, Horlings H, Kreike B, Hayes M, Hauptmann M, Wessels L, De Jong D, Van de Vijver M, Van't Veer LJ and Peterse J: Refinement of breast cancer classification by molecular characterization of histological special types. J Pathol 216: 141-150, 2008.

12. Yates LR, Gerstung M, Knappskog S, Desmedt C, Gundem G, Van Loo P, Aas T, Alexandrov LB, Larsimont D, Davies H, et al: Subclonal diversification of primary breast cancer revealed by multiregion sequencing. Nat Med 21: 751-759, 2015.

13. Nik-ZainalS, VanLooP,WedgeDC,AlexandrovLB,GreenmanCD, Lau KW, Raine K, Jones D, Marshall J, Ramakrishna M, et al: The life history of 21 breast cancers. Cell 149: 994-1007, 2012.

14. Meacham CE and Morrison SJ: Tumour heterogeneity and cancer cell plasticity. Nature 501: 328-337, 2013.

15. Greaves $M$ and Maley CC: Clonal evolution in cancer. Nature 481: 306-313, 2012.

16. Linder D and Gartler SM: Glucose-6-phosphate dehydrogenase mosaicism: Utilization as a cell marker in the study of leiomyomas. Science 150: 67-69, 1965.

17. Lyon MF: Gene Action in the X-chromosome of the Mouse (Mus musculus L.). Nature 190: 372-373, 1961.

18. Wu Y, Basir Z, Kajdacsy-Balla A, Strawn E, Macias V, Montgomery K and Guo SW: Resolution of clonal origins for endometriotic lesions using laser capture microdissection and the human androgen receptor (HUMARA) assay. Fertil Steril 79 (Suppl 1): S710-S717, 2003.

19. Goldstein NS, Vicini FA, Hunter S, Odish E, Forbes S and Kestin LL: Molecular clonality relationships in initial carcinomas, ipsilateral breast failures, and distant metastases in patients treated with breast-conserving therapy: Evidence suggesting that some distant metastases are derived from ipsilateral breast failures and that metastases can metastasize. Am J Clin Pathol 124: 49-57, 2005.

20. Goldstein NS, Vicini FA, Hunter S, Odish E, Forbes S, Kraus D and Kestin LL: Molecular clonality determination of ipsilateral recurrence of invasive breast carcinomas after breast-conserving therapy: Comparison with clinical and biologic factors. Am J Clin Pathol 123: 679-689, 2005.

21. Teixeira MR, Ribeiro FR, Torres L, Pandis N, Andersen JA, Lothe RA and Heim S: Assessment of clonal relationships in ipsilateral and bilateral multiple breast carcinomas by comparative genomic hybridisation and hierarchical clustering analysis. $\mathrm{Br}$ J Cancer 91: 775-782, 2004.

22. Masuda S, Kadowaki T, Kumaki N, Tang X, Tokuda Y, Yoshimura S, Takekoshi S and Osamura RY: Analysis of gene alterations of mitochondrial DNA D-loop regions to determine breast cancer clonality. Br J Cancer 107: 2016-2023, 2012.

23. Lakhani SR, Rakha E, Simpson PT: Invasive lobular carcinoma. In: WHO classification of tumours of the breast. Lakhani SR, Ellis IO, Schnitt SJ, Tan PH, van de Vijver MJ (eds.) International Agency for Research on Cancer, Lyon, 2012.

24. Shin SJ, Desmedt C, Kristiansen G, Reis-Filho JS, Sasano $\mathrm{H}$ : Invasive lobular carcinoma. In: Breast Tumours. WHO Classification of Tumours Editorial Board (ed.) International Agency for Research on Cancer, Lyon, 2019.

25. McCart Reed AE, Kutasovic JR, Nones K, Saunus JM, Da Silva L, Newell F, Kazakoff S, Melville L, Jayanthan J, Vargas AC, et al: Mixed ductal-lobular carcinomas: Evidence for progression from ductal to lobular morphology. J Pathol 244: 460-468, 2018.

26. Tazaki E, Shishido-Hara Y, Mizutani N, Nomura S, Isaka H, Ito H, Imi K, Imoto S and Kamma H: Histopathologcial and clonal study of combined lobular and ductal carcinoma of the breast. Pathol Int 63: 297-304, 2013. 
27. Nakayama Y, Yamaguchi H, Einaga N and Esumi M: Pitfalls of DNA quantification using DNA-binding fluorescent dyes and suggested solutions. PLoS One 11: e0150528, 2016.

28. Einaga N, Yoshida A, Noda H, Suemitsu M, Nakayama Y, Sakurada A, Kawaji Y, Yamaguchi H, Sasaki Y, Tokino T and Esumi M: Assessment of the quality of DNA from various formalin-fixed paraffin-embedded (FFPE) tissues and the use of this DNA for next-generation sequencing (NGS) with no artifactual mutation. PLoS One 12: e0176280, 2017.

29. Schmittgen TD and Livak KJ: Analyzing real-time PCR data by the comparative C(T) method. Nature Protoc 3: 1101-1108, 2008.

30. Stoneking M: Hypervariable sites in the mtDNA control region are mutational hotspots. Am J Hum Genet 67: 1029-1032, 2000.

31. Nakai T, Sakurada A, Endo T, Kobayashi H, Masuda S, Makishima M and Esumi M: Caution for simple sequence repeat number variation in the mitochondrial DNA D-loop to determine cancer-specific variants. Oncol Lett 17: 1883-1888, 2019.

32. Rychlik W, Spencer WJ and Rhoads RE: Optimization of the annealing temperature for DNA amplification in vitro. Nucleic Acids Res 18: 6409-6412, 1990.

33. Hwang ES, DeVries S, Chew KL, Moore DH II, Kerlikowske K, Thor A, Ljung BM and Waldman FM: Patterns of chromosomal alterations in breast ductal carcinoma in situ. Clin Cancer Res 10: 5160-5167, 2004.

34. Huiping C, Sigurgeirsdottir JR, Jonasson JG, Eiriksdottir G, Johannsdottir JT, Egilsson V and Ingvarsson S: Chromosome alterations and E-cadherin gene mutations in human lobular breast cancer. Br J Cancer 81: 1103-1110, 1999.

35. Buerger H, Otterbach F, Simon R, Schäfer KL, Poremba C, Diallo R, Brinkschmidt C, Dockhorn-Dworniczak B and Boecker W: Different genetic pathways in the evolution of invasive breast cancer are associated with distinct morphological subtypes. J Pathol 189: 521-526, 1999.
36. Mohsin SK, O'Connell P, Allred DC and Libby AL: Biomarker profile and genetic abnormalities in lobular carcinoma in situ. Bresast Cancere Res Treat 90: 249-256, 2005.

37. Weigelt B, Geyer FC, Natrajan R, Lopez-Garcia MA, Ahmad AS, Savage K, Kreike B and Reis-Filho JS: The molecular underpinning of lobular histological growth pattern: A genome-wide transcriptomic analysis of invasive lobular carcinomas and grade- and molecular subtype-matched invasive ductal carcinomas of no special type. J Pathol 220: 45-57, 2010.

38. Lopez-Garcia MA, Geyer FC, Lacroix-Triki M, Marchio C and Reis-Filho JS: Breast cancer precursors revisited: Molecular features and progression pathways. Histopathology 57: 171-192, 2010.

39. Abdel-Fatah TMA, Powe DG, Hodi Z, Reis-Filho JS, Lee AHS and Ellis IO: Morphologic and molecular evolutionary pathways of low nuclear grade invasive breast cancers and their putative precursor lesions: Further evidence to support the concept of low nuclear grade breast neoplasia family. Am J Surg Pathol 32: 513-523, 2008.

40. Razavi P, Chang MT, Xu G, Bandlamudi C, Ross DS, Vasan N, Cai Y, Bielski CM, Donoghue MT, Jonsson P, et al: The genomic landscape of endocrine-resistant advanced breast cancers. Cancer Cell 34: 427-438. e426, 2018.

41. Da Silva L, Parry S, Reid L, Keith P, Waddell N, Kossai M, Clarke C, Lakhani SR and Simpson PT: Aberrant expression of E-cadherin in lobular carcinomas of the breast. Am J Surg Pathol 32: 773-783, 2008.

This work is licensed under a Creative Commons Attribution-NonCommercial-NoDerivatives 4.0 International (CC BY-NC-ND 4.0) License. 